\title{
Effect of simple-bundles management vs. guideline-bundles management on elderly patients with septic shock: a retrospective study
}

\author{
Qian Yang', Zhong Wang ${ }^{2}$, Jian Guan $^{1}$ \\ ${ }^{1}$ Emergency Department, First Hospital of Tsinghua University, Beijing, China; ${ }^{2}$ Beijing Tsinghua Changgung Hospital, Beijing, China \\ Contributions: (I) Conception and design: Q Yang; (II) Administrative support: Q Yang, J Guan; (III) Provision of study materials or patients: Q Yang, \\ J Guan; (IV) Collection and assembly of data: Q Yang, Z Wang; (V) Data analysis and interpretation: Q Yang, Z Wang; (VI) Manuscript writing: All \\ authors; (VII) Final approval of manuscript: All authors. \\ Correspondence to: Jian Guan. Emergency Department, First Hospital of Tsinghua University, No.6 1st Block Jiuxianqiao, Beijing 100016, China. \\ Email: jianguan19@tom.com.
}

Background: The objective of this study was to compare the effects of simple-bundles management and
guideline-bundles management on elderly patients with severe sepsis and septic shock.
Methods: This was a single-center retrospective observational study of elderly patients with a hospital
discharge diagnosis of severe sepsis or septic shock from First Hospital of Tsinghua University from January
2014 to December 2018. Management for patients from emergency ward adhered to the simple bundles.
Management for patients from intensive care unit adhered to Surviving Sepsis Campaign Guidelines 2012 .
Data of the two groups were collected to determine the demographic, the source of infection, the baseline
physiology, the Acute Physiology and Chronic Health Evaluation (APACHE) II Scores and the serum lactate
concentration during the in-hospital period and were compared. The 30-, 60-, and 90-day mortality were
compared between the two groups.

Results: One hundred patients came from emergency ward and ninety-eight patients came from intensive care unit met the inclusion criteria. There were no significant differences between the two groups in demographic and clinical characteristics at baseline $(\mathrm{P}>0.05)$. There were no significant differences between the two groups in the APACHE-II scores and the lactate concentration at 6, 24, 72 hours after management and before management $(\mathrm{P}>0.05)$. There were also no significant differences between the two groups in the 30-, 60- and 90-day mortality $(\mathrm{P}>0.05)$.

Conclusions: The level of improvement achieved in the simple-bundles group was similar with that achieved in the guideline-bundles group. Guideline-bundles management, compared with simple-bundles management, might not decrease mortality among elderly patients with severe sepsis and septic shock.

Keywords: Simple-bundles management; guideline-bundles management; elderly patients; severe sepsis; septic shock

Submitted Dec 21, 2020. Accepted for publication Mar 21, 2021.

doi: 10.21037/apm-20-2320

View this article at: http://dx.doi.org/10.21037/apm-20-2320

\section{Introduction}

Based on the current researches, sepsis is defined as the presence of infection together with systemic manifestations of infection, while severe sepsis is defined as sepsis plus sepsis-induced organ dysfunction or tissue hypoperfusion and septic shock is defined as sepsis-induced hypotension persisting despite adequate fluid resuscitation (1).

Septic shock is associated with incremental complications and mortality, and this type of patients are usually admitted to intensive care unit (ICU) and receive bundles treatment 
according to guidelines (1). Nowadays, for many reasons such as hospital bed shortage, bad financial condition of patients or impracticably invasive monitoring and treatment which refused by family members, a lot of patients with severe sepsis and septic shock especially elderly patients weren't admitted to ICU. In our hospital, such kind of patients were admitted to emergency ward and received more simple, noninvasive treatment.

Simple-bundles management has been shown to substantially improve patient outcome in critical illness (2). Simple-bundles management uses a protocol to guide therapy to achieve a predetermined target. The potential benefits of protocol usage arise from reducing variability of medical practice and decreasing errors, thereby improving clinical outcomes and reducing medical costs. Other showed that when guideline-bundles management were implemented in the emergency department, survival outcomes in patients with severe sepsis and septic shock significantly improved (3). However, the comparison of simple-bundles management and guideline-bundles management remains controversial.

In response to this phenomenon, we implemented a single-center retrospective observational study to compare the effect of simple-bundles management with that of guideline-bundles management for elderly patients with severe sepsis and septic shock.

We present the study in accordance with the STROBE reporting checklist (available at http://dx.doi.org/10.21037/ apm-20-2320).

\section{Methods}

\section{Patients}

This was a retrospective observational study of elderly patients ( $\geq 60$ years old) with a hospital discharge diagnosis of either severe sepsis or septic shock (ICD-10) from First hospital of Tsinghua university from January 2014 to December 2018.

All elderly patients meeting severe sepsis or septic shock clinical criteria in the dataset within the emergency ward and the ICU were included in our study. The inclusion criteria for severe sepsis were suspected or confirmed infection, two or more general and inflammatory variables, and evidence of organ dysfunction. General and inflammatory variables included the following: body temperature $>38.3$ or $<36^{\circ} \mathrm{C}$; heart rate $>90$ beats/min; tachypnea; altered mental status; significant edema or positive fluid balance $(20 \mathrm{~mL} / \mathrm{kg}$ over $24 \mathrm{~h}$ ); plasma glucose $>140 \mathrm{mg} / \mathrm{dL}$ or $7.7 \mathrm{mmol} / \mathrm{L}$ in the absence of diabetes; white blood cell count $>12,000 / \mathrm{mm}^{3}$ or $<4,000 / \mathrm{mm}^{3}$ or a bandemia of $>10 \%$; plasma $\mathrm{C}$-reactive protein or procalcitonin more than two SD above the normal value. Organ dysfunction was defined as a new-onset $\mathrm{PaO}_{2} / \mathrm{FiO}_{2}$ $<300$, urine output $<0.5 \mathrm{~mL} / \mathrm{kg} / \mathrm{h}$ for at least 2 hours despite adequate fluid resuscitation, a new-onset creatinine increase $>0.5 \mathrm{mg} / \mathrm{dL}$ or $44.2 \mu \mathrm{mol} / \mathrm{L}$, a new-onset INR $>1.5$ or APTT $>60 \mathrm{~s}$ in the absence of anticoagulants, absent bowel sounds, a new-onset platelet count $<100,000 / \mu \mathrm{L}$, or a new-onset plasma total bilirubin $>4 \mathrm{mg} / \mathrm{dL}$ or $70 \mu \mathrm{mol} / \mathrm{L}$. Septic shock was defined as severe sepsis plus lactate $\geq 4 \mathrm{mmol} / \mathrm{L}$ and/or systolic blood pressure (SBP) $<90 \mathrm{mmHg}$ or mean arterial pressure (MAP) $<65 \mathrm{mmHg}$ after an intravenous fluid load of at least $20 \mathrm{~mL} / \mathrm{kg}$ over 60 minutes. Patients were excluded if the following criteria were present: any primary diagnosis other than sepsis, active malignancy (diagnosed either before or during admission) and being transferred from another hospital.

\section{Treatment}

Management on patients from ICU completely adhered to Surviving Sepsis Campaign (SSC) Guidelines 2012 (1): (I) early goal-directed therapy (EGDT) during the first 6 hours, which aimed to achieve the goals of (i) a central venous pressure (CVP) $8-12 \mathrm{mmHg}$, (ii) a $\mathrm{MAP} \geq 65 \mathrm{mmHg}$, (iii) a urine output $\geq 0.5 \mathrm{~mL} / \mathrm{kg} / \mathrm{h}$ and (iv) a superior vena cava oxygenation saturation ( $\mathrm{ScvO} 2) \geq 70 \%$; (II) endotracheal intubation and invasive mechanical ventilation when necessary; (III) lactate levels measured within the first 3 hours; (IV) blood cultures prior to antibiotic administration; (V) broad spectrum antibiotics administered within the first 3 hours; (VI) a minimum of $30 \mathrm{~mL} / \mathrm{kg}$ of crystalloid fluids administered within the first 3 hours; (VII) vasopressors applied to maintain a MAP $\geq 65 \mathrm{mmHg}$ for hypotension that does not respond to initial fluid resuscitation.

Management on patients from emergency ward adhered to the simple bundles was as following. The simple-bundles were defined as the following: (I) continuous monitoring of vital signs non-invasively; (II) placement of the peripherally inserted central venous catheter (PICC) instead of the central venous catheter to administer intravenous fluids and vasopressors; (III) application of the non-invasive ventilation, sputum aspiration, clapping back sputum elimination or vibration sputum elimination instead of invasive mechanical ventilation; (IV) lactate levels measured within the first 3 hours; (V) blood cultures prior to antibiotic administration; (VI) broad spectrum antibiotics administered within the first 3 hours; (VII) a minimum of $30 \mathrm{~mL} / \mathrm{kg}$ of crystalloid fluids 
administered within the first 3 hours; (VIII) vasopressors applied to maintain a MAP $\geq 65 \mathrm{mmHg}$ for hypotension that does not respond to initial fluid resuscitation.

\section{Measures of clinical outcomes}

Data of the above two groups were collected to determine the demographic, the source of infection, the baseline physiology, the Acute Physiology and Chronic Health Evaluation (APACHE) II Scores and the serum lactate concentration during the in-hospital period and were compared. The 30-, 60-, and 90-day mortality were compared between the two groups.

\section{Statistical analysis}

The statistical software SPSS 22.0 (Copyright IBM Corporation and other) was used to analyze the data. Continuous variables were presented as mean \pm SD (Standard Deviation). Categorical variables were reported as proportions. The Shapiro-Wilk test was used to assess the normality of continuous data. The Mann-Whitney U-test was used to compare between-group differences and the Wilcoxon test was used to compare intragroup differences for continuous variables. The Fisher's exact test was used to compare between-group differences for categorical variables. In all cases, two-side $\mathrm{P}<0.05$ was taken as the level of significance.

\section{Ethical statement}

The study was approved by ethics board of First Hospital of Tsinghua University (No.: 2019(04)). The study was conducted in accordance with the 1964 Helsinki declaration and its later amendments or comparable ethical standards.

All patients from emergency ward whose relatives had provided informed written refusal refused great intensity invasive monitoring and treatment such as endotracheal intubation, invasive mechanical ventilation, cardiac massage and defibrillation. All patients from ICU whose relatives had provided informed written consent agreed to great intensity invasive monitoring and treatment as mentioned above.

\section{Results}

One hundred patients came from emergency ward and ninety-eight patients came from ICU meeting the inclusion criteria were enrolled into the study. The baseline data of the two groups were presented in Table 1. The lungs were the most common source of infection in both groups. There were no significant differences between the two groups in demographic and clinical characteristics at baseline.

The APACHE-II scores in the two groups before and after management were presented in Table 2. The APACHE-II scores in the simple-bundles group at 6 hours after management $(Z=-7.631, P<0.001), 24$ hours after management $(Z=-8.196, P<0.001)$ and 72 hours after management $(Z=-7.832, P<0.001)$ significantly decreased comparing with before management; and the APACHEII scores at 24 hours after management significantly decreased comparing with 6 hours after management $(\mathrm{Z}=-8.223, \mathrm{P}<0.001)$; the APACHE-II scores at 72 hours after management significantly decreased comparing with 24 hours after management $(Z=-7.530, P<0.001)$. The APACHE-II scores in the guideline-bundles group manifested a similar improvement $(6$ hours after management $v s$. before management: $\mathrm{Z}=-7.655, \mathrm{P}<0.001$; 24 hours after management $v$ s. before management: $\mathrm{Z}=-8.148, \mathrm{P}<0.001 ; 72$ hours after management $v s$. before management: $Z=-7.731, P<0.001 ; 24$ hours after management vs. 6 hours after management: $Z=-7.885$, $\mathrm{P}<0.001 ; 72$ hours after management vs. 24 hours after management: $Z=-7.273, P<0.001)$. There were no significant differences between the two groups in the APACHE-II scores at 6 hours, 24 hours, 72 hours after management and before management $(\mathrm{P}>0.05)$.

The lactate concentration in the two groups before and after management were presented in Table 3. The lactate concentration in the simple-bundles group at 6 hours after management $(Z=-7.331, P<0.001), 24$ hours after management $(\mathrm{Z}=-7.886, \mathrm{P}<0.001)$ and 72 hours after management $(\mathrm{Z}=-7.983, \mathrm{P}<0.001)$ significantly decreased comparing with before management; and the lactate concentration at 24 hours after management significantly decreased comparing with 6 hours after management $(\mathrm{Z}=-7.973, \mathrm{P}<0.001)$; the lactate concentration at 72 hours after management significantly decreased comparing with 24 hours after management $(\mathrm{Z}=-7.736, \mathrm{P}<0.001)$. The lactate concentration in the guideline-bundles group manifested a similar improvement (6 hours after management $v$ s. before management: $Z=-7.364, P<0.001 ; 24$ hours after management vs. before management: $Z=-7.533, \mathrm{P}<0.001 ; 72$ hours after management $v s$. before management: $Z=-7.874, \mathrm{P}<0.001$; 24 hours after management $v s .6$ hours after management: $\mathrm{Z}=-7.515, \mathrm{P}<0.001 ; 72$ hours after management $v s .24$ hours 
Table 1 Baseline data of two groups

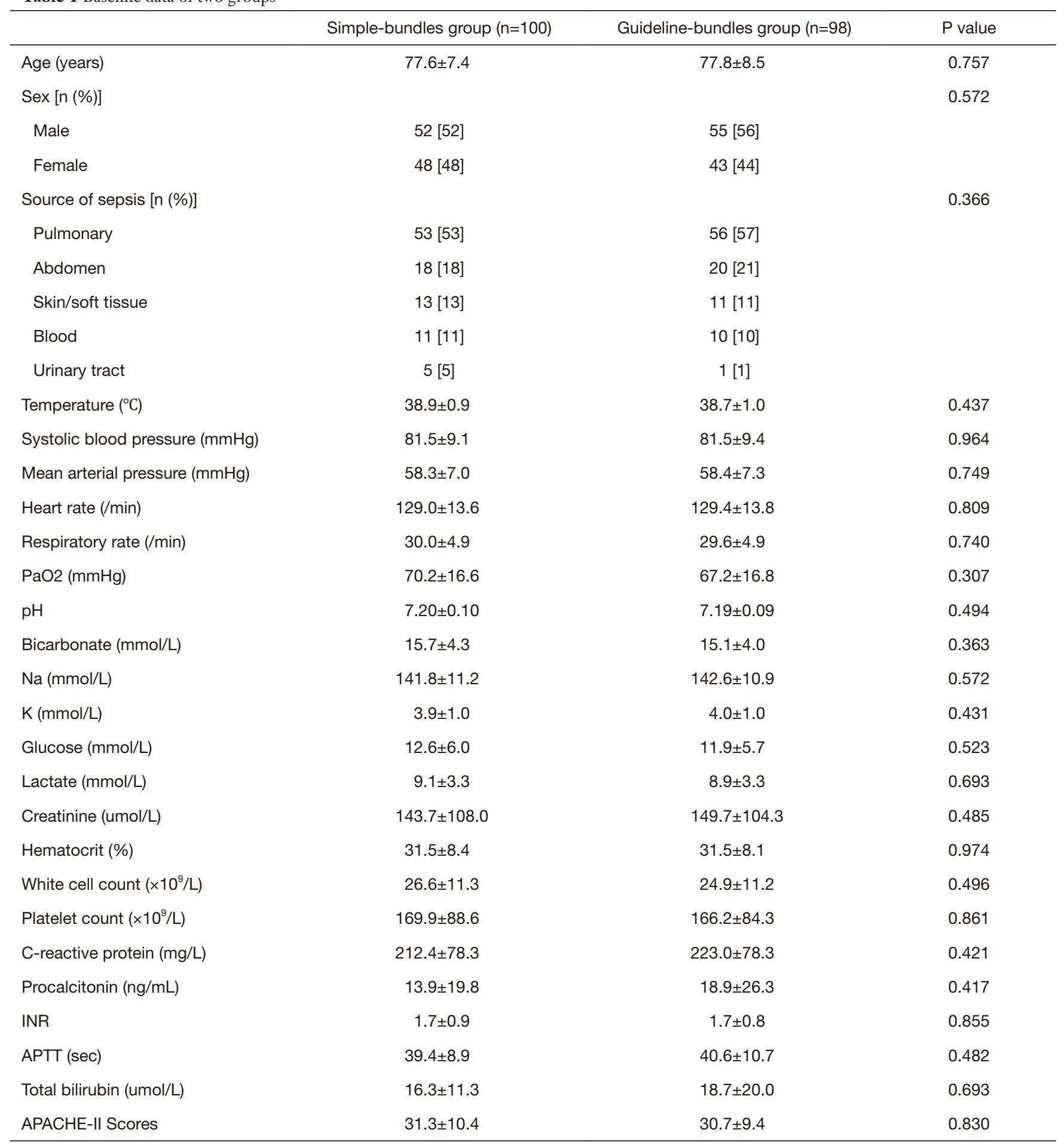

INR, international normalized ratio; APTT, activated partial thromboplastin time. 
Table 2 APACHE-II scores in two groups before and after management

\begin{tabular}{lcccc}
\hline & Before & 6 hours after & 24 hours after & 72 hours after \\
\hline Simple-bundles group $(n=100)$ & $31.3 \pm 10.4$ & $27.4 \pm 11.5$ & $20.9 \pm 10.5$ & $16.3 \pm 10.0$ \\
Guideline-bundles group $(n=98)$ & $30.7 \pm 9.4$ & $26.3 \pm 10.4$ & $20.0 \pm 10.2$ & $15.1 \pm 9.5$ \\
$P$ value & 0.830 & 0.616 & 0.475 & 0.331 \\
\hline
\end{tabular}

Table 3 Lactate concentration in two groups before and after management

\begin{tabular}{lcccc}
\hline & Before & 6 hours after & 24 hours after & 72 hours after \\
\hline Simple-bundles group $(n=100)$ & $9.1 \pm 3.3$ & $8.2 \pm 3.6$ & $6.2 \pm 3.6$ & $4.4 \pm 3.4$ \\
Guideline-bundles group $(n=98)$ & $8.9 \pm 3.3$ & $8.0 \pm 3.5$ & $6.2 \pm 3.5$ & $4.3 \pm 3.3$ \\
P value & 0.693 & 0.708 & 0.991 & 0.969 \\
\hline
\end{tabular}

Table 4 Mortality for two groups

\begin{tabular}{lccc}
\hline & 30-day mortality [n (\%)] & 60-day mortality [n (\%)] & 90-day mortality [n (\%)] \\
\hline Simple-bundles group $(\mathrm{n}=100)$ & $29(29.0)$ & $33(33.0)$ & $36(36.0)$ \\
Guideline-bundles group $(\mathrm{n}=98)$ & $27(27.6)$ & $32(32.7)$ & $36(36.7)$ \\
P value & 0.875 & 0.959 & 0.915 \\
\hline
\end{tabular}

after management: $Z=-7.827, P<0.001)$. There were no significant differences between the two groups in the lactate concentration at $6,24,72$ hours after management and before management $(\mathrm{P}>0.05)$.

The follow-up rate of the two groups was $100 \%$. The mortality for the two groups were presented in Table 4. The 30-, 60- and 90-day mortality for the simple-bundles group were $29.0 \%, 33.0 \%$ and $36.0 \%$, respectively. And that for the guideline-bundles group were $27.6 \%, 32.7 \%$ and $36.7 \%$, respectively. The 30-day, 60 -day and 90-day mortality for the simple-bundles group did not differ significantly from that for the guideline-bundles group $(\mathrm{P}>0.05)$.

\section{Discussion}

The annual incidence of severe sepsis and septic shock in adults is reported to range from 56 to 91 per 100,000 population (4). The complication rate and mortality remained high for many years (5-7).

The aging of population has been a global phenomenon. The elderly people are the predisposing group of severe sepsis and septic shock because of higher rates of chronic disease and worse functional status. The number of elderly patients with severe sepsis and septic shock is increasing year by year, therefore, we implemented this study.

In our study, the guideline-bundles management led to a significant improvement in the condition of patients from ICU, this result is similar with those of previous studies $(8,9)$. It is worth noting that the simple-bundles group also manifested significant improvements in the APACHE-II scores and the lactate concentration; however, the subsequent APACHE-II scores, the subsequent lactate concentration and the all-cause mortality for the simplebundles group did not differ significantly from that for the guideline-bundles group.

It was found that EGDT did not result in lower mortality than usual care and EGDT as a packaged protocol of care is not superior to usual care, for example, three largescale, multicenter, randomized, controlled trials from the United States [Protocolized Care for Early Septic Shock (ProCESS)] (10), Australasia [Australasian Resuscitation in Sepsis Evaluation (ARISE)] (11), and the United Kingdom [Protocolised Management in Sepsis (ProMISe)] (12). These results are similar to those of our study. The three trials gave one reason as the following (10-12): Nowadays, many hospitals have achieved levels of in-hospital survival in patients receiving usual care that were similar to those achieved with EGDT in the earlier study for patients who 
were identified early and received intravenous antibiotics and adequate fluid resuscitation; general improvement in the provision of care for sepsis and septic shock during the past decade may have helped lower the overall mortality and reduced the marginal benefit of alternative resuscitation strategies. It may also be one of the many reasons why we reached the above conclusion.

It should be emphasized that the simple-bundles management in our study is different from the "usual care" in the previous studies. Our approach is more noninvasive and simple because of placement of PICC and unemployment of endotracheal intubation and invasive mechanical ventilation. The simple-bundles group manifested significant reduction in the APACHE-II scores and the lactate concentration under management of the above approach, and the all-cause mortality for the simple-bundles group is similar to those of previous studies (8-17). It is suggested that there may be no absolute causal relationship between curative effect and therapeutic intensity; our study showed the benefit of the simple-bundles management and it can help to alleviate patients' pain and save medical resources.

It is reported that the insertion of central venous catheter is not completely safe and accompanied with the risk of complications, such as heart arrhythmias, artery punctures, hematoma, pneumothorax and infections at the location of the insertion (18). Administration of vasopressors through peripheral intravenous catheters may result in extravasation and local tissue injury (19). It is showed in some literatures that PICC reduces cost because of longer placement time and decreases the risk for catheter occlusion, pneumothorax and infections $(20,21)$. In our study, PICC was placed to administer intravenous fluids and vasopressors, it may be one of the reasons that our study achieved the above results. The main limitation of this study was that there should be clinically heterogeneous like financial condition of patients in this study, which might affect our conclusion.

In our single-center, retrospective study, the levels of improvement achieved in the simple-bundles group were similar with that achieved in the guideline-bundles group for elderly patients with severe sepsis and septic shock. Guideline-bundles management, as compared with simplebundles management, did not decrease mortality among elderly patients with severe sepsis and septic shock.

\section{Acknowledgments}

We would like to thank Dr. Huang Hua for his help in polishing the language of our paper.
Funding: None.

\section{Footnote}

Reporting Checklist: The authors have completed the STROBE reporting checklist. Available at http://dx.doi. org/10.21037/apm-20-2320

Data Sharing Statement: Available at http://dx.doi. org/10.21037/apm-20-2320

Conflicts of Interest: All authors have completed the ICMJE uniform disclosure form (Available at http://dx.doi. org/10.21037/apm-20-2320). The authors have no conflicts of interest to declare.

Ethical Statement: The authors are accountable for all aspects of the work in ensuring that questions related to the accuracy or integrity of any part of the work are appropriately investigated and resolved. The study was approved by ethics board of First Hospital of Tsinghua University (No.: 2019(04)). The study was conducted in accordance with the 1964 Helsinki declaration and its later amendments or comparable ethical standards. All patients from emergency ward whose relatives had provided informed written refusal refused great intensity invasive monitoring and treatment such as endotracheal intubation, invasive mechanical ventilation, cardiac massage and defibrillation. All patients from ICU whose relatives had provided informed written consent agreed to great intensity invasive monitoring and treatment as mentioned above.

Open Access Statement: This is an Open Access article distributed in accordance with the Creative Commons Attribution-NonCommercial-NoDerivs 4.0 International License (CC BY-NC-ND 4.0), which permits the noncommercial replication and distribution of the article with the strict proviso that no changes or edits are made and the original work is properly cited (including links to both the formal publication through the relevant DOI and the license). See: https://creativecommons.org/licenses/by-nc-nd/4.0/.

\section{References}

1. Dellinger RP, Levy MM, Rhodes A, et al. Surviving Sepsis Campaign: international guidelines for management of severe sepsis and septic shock, 2012. Intensive Care Med 2013;39:165-228. 
2. Burns SM, Earven S, Fisher C, et al. Implementation of an institutional program to improve clinical and financial outcomes of mechanically ventilated patients: one-year outcomes and lessons learned. Crit Care Med 2003;31:2752-63.

3. Rivers E, Nguyen B, Havstad S, et al. Early goal-directed therapy in the treatment of severe sepsis and septic shock. N Engl J Med 2001;345:1368-77.

4. Jawad I, Luksic I, Rafnsson SB. Assessing available information on the burden of sepsis: global estimates of incidence, prevalence and mortality. J Glob Health 2012;2:010404.

5. Levy MM, Artigas A, Phillips GS, et al. Outcomes of the Surviving Sepsis Campaign in intensive care units in the USA and Europe: a prospective cohort study. Lancet Infect Dis 2012;12:919-24.

6. Kaukonen KM, Bailey M, Suzuki S, et al. Mortality related to severe sepsis and septic shock among critically ill patients in Australia and New Zealand, 2000-2012. JAMA 2014;311:1308-16.

7. Cuthbertson BH, Elders A, Hall S, et al. Mortality and quality of life in the five years after severe sepsis. Crit Care 2013;17:R70.

8. Sivayoham N, Rhodes A, Jaiganesh T, et al. Outcomes from implementing early goal-directed therapy for severe sepsis and septic shock : a 4-year observational cohort study. Eur J Emerg Med 2012;19:235-40.

9. Milano PK, Desai SA, Eiting EA, et al. Sepsis Bundle Adherence Is Associated with Improved Survival in Severe Sepsis or Septic Shock. West J Emerg Med 2018;19:774-81.

10. ProCESS Investigators, Yealy DM, Kellum JA, et al. A randomized trial of protocol-based care for early septic shock. N Engl J Med 2014;370:1683-93.

11. ARISE Investigators, ANZICS Clinical Trials Group, Peake SL, et al. Goal-directed resuscitation for patients with early septic shock. N Engl J Med 2014;371:1496-506.

12. Mouncey PR, Osborn TM, Power GS, et al. Trial of early, goal-directed resuscitation for septic shock. N Engl J Med
2015;372:1301-11.

13. Jones AE, Shapiro NI, Trzeciak S, et al. Lactate clearance vs central venous oxygen saturation as goals of early sepsis therapy: a randomized clinical trial. JAMA 2010;303:739-46.

14. Hernandez G, Ospina-Tascon GA, Damiani LP, et al. Effect of a Resuscitation Strategy Targeting Peripheral Perfusion Status vs Serum Lactate Levels on 28-Day Mortality Among Patients With Septic Shock: The ANDROMEDA-SHOCK Randomized Clinical Trial. JAMA 2019;321:654-64.

15. Labelle A, Juang P, Reichley R, et al. The determinants of hospital mortality among patients with septic shock receiving appropriate initial antibiotic treatment*. Crit Care Med 2012;40:2016-21.

16. Pavon A, Binquet C, Kara F, et al. Profile of the risk of death after septic shock in the present era: an epidemiologic study. Crit Care Med 2013;41:2600-9.

17. McEvoy C, Kollef MH. Determinants of hospital mortality among patients with sepsis or septic shock receiving appropriate antibiotic treatment. Curr Infect Dis Rep 2013;15:400-6.

18. Hodzic S, Golic D, Smajic J, et al. Complications Related to Insertion and Use of Central Venous Catheters (CVC). Med Arch 2014;68:300-3.

19. Loubani OM, Green RS. A systematic review of extravasation and local tissue injury from administration of vasopressors through peripheral intravenous catheters and central venous catheters. J Crit Care 2015;30:653.e9-17.

20. Johansson E, Hammarskjold F, Lundberg D, et al. Advantages and disadvantages of peripherally inserted central venous catheters (PICC) compared to other central venous lines: a systematic review of the literature. Acta Oncol 2013;52:886-92.

21. Chiba H, Endo K, Izumiyama Y, et al. Usefulness of a peripherally inserted central catheter for total parenteral nutrition in patients with inflammatory bowel disease. Nihon Shokakibyo Gakkai Zasshi 2017;114:1639-48.
Cite this article as: Yang Q, Wang Z, Guan J. Effect of simplebundles management $v s$. guideline-bundles management on elderly patients with septic shock: a retrospective study. Ann Palliat Med 2021;10(5):5198-5204. doi: 10.21037/apm-20-2320 